\title{
A Comprehensive HPV-STI NGS Assay for Detection of 29 HPV Types and 14 non- HPV Sexually Transmitted Infections
}

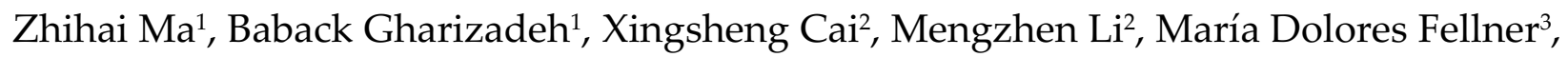
Jorge Alejandro Basiletti ${ }^{3}$, Rita Mariel Correa ${ }^{3}$, María Celeste Colucci ${ }^{3}$, Gabriela Baldoni ${ }^{4}$, Martín Vacchino ${ }^{4}$, Patricia Galarza ${ }^{4}$, María Alejandra Picconi ${ }^{3}$, Chunlin Wang ${ }^{1}$

${ }^{1}$ Chapter Diagnostics, 1455 Adams Drive, Menlo Park, CA 94025, USA

${ }^{2}$ Maijing Gene Medical Technology, No. 9, Spiral 4th Road, International Biological Island, Guangzhou, China

${ }^{3}$ Oncogenic Viruses Service, National and Regional HPV Reference Laboratory, National Institute of Infectious Diseases-ANLIS “Dr. Malbrán”, Av. Velez Sarsfield 563, C1282AFF Buenos Aires, Argentina

"Sexually Transmitted Diseases Service, National Reference Laboratory for STDs, National Institute of Infectious Diseases - ANLIS “Dr Malbrán”, Av. Velez Sarsfield 563, C1282AFF Buenos Aires, Argentina

Corresponding Author:

Chunlin Wang, Ph.D.

Chapter Diagnostics Inc.

1455 Adams drive

Menlo Park, CA 94025, USA

chunlin@chapterdx.com 


\begin{abstract}
Sexually transmitted infections (STIs) are prevalent throughout the world and impose a significant burden on individual health and public health systems. Missed diagnosis and late treatment of STIs can lead to serious complications such as infertility and cervical cancer. Although sexually transmitted co-infections are common, most commercial assays target one or a few STIs. The HPV-STI ChapterDx Next Generation Sequencing (NGS) assay detects and quantifies $29 \mathrm{HPVs}$ and 14 other STIs in a single-tube and single-step PCR reaction and can be applied to tens to thousands of samples in a single sequencing run. The assay was evaluated in this study, and the limit of detection was $100 \%$ at 50 copies for all targets, and 100\%, 96\%, 88\% at 20 copies for 34, 8, and 1 target, respectively. The performance of this assay has been compared to Roche cobas HPV test, showing an overall agreement of $97.5 \%$ for hr-HPV, and 98.5\% for both, HPV16 and HPV18. The assay also detected all HPV-infected CIN2/3 with 100\% agreement with Roche cobas HPV results. Moreover, several co-infections with non-HPV STIs, such as C. trachomatis, $T$. vaginalis, M. genitalium, and HSV2 were identified. The ChapterDx HPV-STI NGS assay is a user-friendly, easy to automate and cost-efficient assay, which provides accurate and comprehensive results for a wide spectrum of HPVs and STIs.
\end{abstract}

\title{
Keywords
}

Human papillomavirus (HPV), Sexually transmitted infections (STI), HPV genotyping, Next generation sequencing (NGS), Sexually transmitted disease (STD), Cervical cancer, Chlamydia trachomatis, Neisseria gonorrhoeae, Treponema pallidum, Trichomonas vaginalis 


\section{INTRODUCTION}

Sexually transmitted infections (STIs) are prevalent globally and World Health Organization (WHO) estimates that annually there are 376 million new cases of four curable STIs: Chlamydia trachomatis, Neisseria gonorrhoeae, Treponema pallidum (syphilis), and Trichomonas vaginalis. Prevalence of several viral STIs is similarly high with an estimated 500 million people infected with herpes simplex type 2 (HSV2), and approximately 291 million women harboring human papillomaviruses (HPV) (1). STI prevalence varies by region, gender. STI epidemics cause a profound impact on physical and psychological health worldwide $[1,2]$.

HPVs are a large group of viruses with more than 230 completely characterized types, and new HPV types being continuously found [3]. Among them, 40 types known to infect human anogenital tract are grouped into high risk (hr)-HPV and low risk (lr)-HPV types based on their oncogenic potential $[4,5]$. Hr-HPVs are the causative agents of cervical cancer, and have been detected in $99.7 \%$ of cervical cancers [6, 7]. Fourteen hr-HPV types are considered to cause most cervical cancers worldwide. Nevertheless, other hr-types have also been reported to cause cervical cancer $[4,5]$. HPV oncogenic potential is highly type-dependent as some types are more oncogenic. Many studies have shown that extended genotyping information holds important clinical value for patient management and triage of HPV positive women [8-10].

HPV viral load quantification has been shown to be a predictor of infection persistence or clearance [11]. It has also been reported that HPV viral load is associated with risk of persistent infection and precancer [12-15], indicating viral load as a candidate risk marker [16]. Currently, viral load is measured mainly by quantitative polymerase chain reaction (qPCR) on a limited number of hr-HPV types such as HPV16 and HPV18. An assay that can detect and quantify many HPVs in a single-tube PCR reaction is a needed tool for association studies of viral load and precancer.

Besides HPVs, several bacteria and viruses infect human anogenital area. Earlier studies indicated that non-HPV STIs could facilitate the entry of HPV virions into host cells, change in the immunological response pathways, and decrease the host's ability to clear HPV infection [16-18]. Co-infections between hr-HPVs and non-HPV STIs such as Chlamydia trachomatis, Neisseria gonorrhoeae, Trichomonas vaginalis, Ureaplasma urealyticum, and HSV2 have been reported to be associated with HPV persistence, cervical dysplastic and neoplastic lesion [19-26]. Co-infections between HPVs and non-HPV STIs are widespread. Since most commercial kits detect one or two STIs, multiple tests are required for comprehensive STI identification. 
This work presents the evaluation of the ChapterDx HPV-STI NGS assay (NGS assay in short) that amplifies 29 HPVs and 14 STIs (Table 1) with species/type-specific primers in a single-tube and single-step PCR reaction followed by NGS (Fig. 1). This assay can detect and quantify these 43 targets from tens to thousands of samples in a single Illumina sequencing run (depending on the sequencing platform and sequencing kit).

\section{MATERIALS AND METHODS}

Clinical HPV screening specimens: A cohort of 274 blind samples that had previously been analyzed by conventional cytology/histology and Roche cobas HPV Test (Roche, Pleasanton, CA, USA) was selected for this study. Within this cohort, 102, 101 and 57 samples were negative for intraepithelial lesions or malignancy (NILM), low-grade squamous intraepithelial lesion (LSIL) and high- grade squamous intraepithelial lesion (HSIL), respectively; and five were cancer and nine samples had no cytology results. The available histological results for this cohort were: cervical intraepithelial neoplasia grade 1 (CIN1) $(\mathrm{N}=4), \mathrm{CIN} 2(\mathrm{~N}=1)$ and CIN3 $(\mathrm{N}=58), 3$ squamous cell carcinoma (SCC) and 2 adenocarcinoma (AdC). HPV samples were shipped from Oncogenic Viruses Service, National and Regional HPV Reference Laboratory, National Institute of Infectious DiseasesANLIS “Dr. Malbrán”, Buenos Aires, Argentina to Chapter Diagnostics in Menlo Park, California, USA, for comprehensive HPV-STI analysis.

ChapterDx HPV-STI NGS kit: ChapterDx HPV-STI NGS kit targets 19 hr-HPVs, 10 lrHPVs, 14 non-HPV STIs, and GAPDH as internal control. Table 1 shows the HPV genotypes and non-HPV STIs covered by the kit.

Controls for analytical performance: A set of 43 synthetic control DNA fragments for all targets were synthesized by Integrated DNA Technologies (Coralville, IA). They were pooled and diluted into 100, 50 and 20 copies per vial in a final volume of $5 \mu$ l. Each pool was spiked with $10 \mathrm{ng}$ human genomic DNA. The pools were amplified and sequenced in 23 replicates for 100 copies and 24 replicates for 50 and 20 copies to assess the limit of detection (LoD) of the NGS assay. The acceptable consensus for an assay's LoD at the lowest concentration is $\geq 95 \%$ for at which $19 / 20$ replicates are positive [27].

DNA extraction and PCR: Genomic DNA extraction for 274-sample cohort were performed by Roche cobas HPV Test (cobas for short) platform. For genotyping 75-sample cohort, DNA extraction was performed by Qiagen EZ1 virus kit (Qiagen, Hilden, Germany). All the specimens were measured by Qubit 3 (ThermoFisher, CA, USA) to monitor the presence and concentration of DNA prior to PCR. One-step multiplex PCR was performed in $15 \mu \mathrm{l}$ final volume in a 96-well plate on a Veriti thermocycler (ThermoFisher, CA, USA). The PCR reaction consisted of target-specific primers (29 HPV, 14 STI and 
internal control), barcoded universal primers, sample DNA, DNA polymerase, dNTPs and PCR buffer. The PCR conditions were according the ChapterDx HPV-STI NGS kit instructions.

\section{Library preparation and next-generation sequencing}

After PCR, the amplified products were pooled into a $1.5 \mathrm{ml}$ tube (or a $15 \mathrm{ml}$ tube depending on the sample size). A portion of the pooled amplicons was purified with SPRIbeads (Beckman Coulter, CA, USA) according to the manufacturer's instructions. The purified pooled amplicons concentration was measured on a Qubit fluorometer, and the concentration was adjusted for sequencing according to Illumina library preparation instructions. The library was sequenced on an Illumina MiniSeq platform using an Illumina Mid-Output sequencing kit (Illumina, CA, USA). The workflow is depicted in Figure 1.

\section{Sequence data analysis software and interpretation}

1) Decoding: sequencing reads (FASTQ format) for forward and reverse reads and forward and reverse indexing reads are input in ChapterDx Analysis Software. The software assigns sequencing reads for a sample based on the sequence of both forward and reverse index reads with no mismatch; 2) Mapping: sequencing reads are mapped onto reference sequences using the Smith-Waterman algorithm with options as nucleotide match reward is 1 , nucleic mismatch penalty is -3 , cost to open a gap is 5 , and cost to extend a gap is 2. Only the alignment of best match is kept for each sequencing read if the alignment score is beyond 60. Alignments of paired reads with identical reference sequences are kept. Reads are labeled to the reference based on their corresponding alignments; 3) Genotyping: a sample is called positive for a species/type when there are more reads than the preset cutoff reads labelled with the corresponding reference.

NGS detection threshold: Based on ChapterDx HPV-STI NGS sequence data from WHO LabNet international proficiency HPV panel [28], the threshold for positive samples were set at 3 reads. Samples below the threshold were considered negative.

Statistical analysis: The HPV and STI species/types frequencies, cytology and histology were calculated by Microsoft excel. Overall agreement for $14 \mathrm{hr}-\mathrm{HPV}, \mathrm{HPV} 16,18$ and other 12 HPVs between ChapterDx HPV-STI NGS and Roche cobas HPV assays were calculated by Cohen's Kappa coefficients with 95\% confidence intervals (CIs). The genotyping comparison between ChapterDx HPV-STI NGS and Genomica Clart assays were calculated by Microsoft excel. 


\section{RESULTS}

\section{Analytical performance of the assay}

To evaluate the analytical performance and sensitivity of the assay, a set of 43 synthetic control DNA fragment pools of 100 copies (23 replicates), 50 copies (24 replicates), 20 copies (24 replicates) and 0 copies (blank) spiked with $10 \mathrm{ng}$ human genomic DNA were analyzed to determine the LoD. 100 copies and 50 copies control DNA were detected in all replicates. For the 20 copies pool replicates, the detection rate was 100\% (24/24) for 34 targets, 95.8\% (23/24) for HPV6, 11, 39, 42, 43, 45, 56 and 83 (8 targets) and 87.5\% (21/24) for Mycoplasma genitalium (Table 2a and 2b). The lowest concentration LoD is recommended at $\geq 95 \%$ (for 19/20 replicates) [27], therefore, the LoD was determined at 20 copies for all targets except for Mycoplasma genitalium. There was no sequencing read for the blank sample.

\section{Agreement between ChapterDx HPV-STI NGS assay and Roche cobas HPV Test in cervical samples with cyto-histological diagnosis}

To evaluate the agreement with the Roche cobas HPV test, 274 clinical samples were analyzed by ChapterDx HPV-STI NGS assay. Out of 274 samples, the results for 267 samples agreed with those by cobas HPV test for $14 \mathrm{hr}-\mathrm{HPV}$ s. Among those 267 samples, 241 were positive and 26 were negative for $14 \mathrm{hr}-\mathrm{HPVs}$. The overall agreement for $14 \mathrm{hr}-\mathrm{HPVs}$ between both methods was $97.5 \%$ with kappa 0.867. The CIN1 (n=4), CIN2/3 (n=59) were positive for hr-HPV by both cobas and the NGS assays (Table 3). Of the 5 cancer samples, one squamous carcinoma sample and one adenocarcinoma sample were negative for 14 hr-HPV by both cobas and the NGS assay. The adenocarcinoma sample was positive for HPV44, 81 by the NGS assay, which was confirmed by reverse line blot assay [29].

The overall agreement for HPV16 between cobas and the NGS assays was 98.5\% (270/274) with a kappa value of 0.967 (Table 4a). Two of the HPV16 samples were scored negative. However, one and two HPV16 reads were detected in each of these two samples, respectively. The overall agreement for HPV18 was $98.5 \%$ (70/274) with a kappa value of (0.901) and the overall agreement for other HPVs was 96\% (263/274) with kappa value of 0.906 . For the other HPVs, two HPV samples were also detected by the NGS assay as HPV52 and HPV31/52, respectively, but were below the NGS detection threshold and scored negative. Of the $29 \mathrm{HPV}$ targets in the NGS panel, 28 were detected among the samples analyzed (except for HPV73). In this cohort, co-infections between 2 and 12 HPVs were detected in 146 samples (shown in Table 4b).

In the 274-sample cohort, ChapterDx HPV-STI NGS assay detected a total frequency of 205 non-HPV STIs, of which U. parvum (32.1\%) and M. hominis (25.2\%) comprised the majority of the non-HPV STIs (Table 5a). 52.1\% (143/274) of the samples were positive for 
7 out of 14 non-HPV STIs, harboring a range of 1-4 STIs per sample (Table 5b). HPV and non-HPV STIs co-infections were observed in 135/274 (49.3\%) of the samples. Table 6 lists results by Roche cobas assay and the NGS assay for 9 samples with co-infections of multiple HPVs and non-HPV STIs, demonstrating detection of HPV and non-HPV STIs by the NGS assay.

In this study, 192 positive samples were sequenced per Illumina Mid-Output sequencing kit on Illumina MiniSeq platform. Table S1 lists the estimated number of samples for different Illumina sequencing platforms and kits.

\section{Discussion}

In the present study, the performance of ChapterDx HPV-STI NGS assay was compared to Roche cobas assay. Forty-three control DNA pools of 100 and 50 copies were detected in all replicates and for 20 copies replicates, $M$. genitalium had an LoD below 95\%. The lower detection rate for $M$. genitalium could be due to synthetic error in control DNA, which affects amplification efficiency. In this study, HPV52 control DNA fragment had to be re-synthesized due to multiple errors found in primer regions.

The NGS and cobas assays showed a high overall agreement, which indicates a good performance of the NGS assay on a set of well-studied samples; nevertheless, a larger study is required based on Meijer's criteria [30] for further clinical evaluation. In this 274sample cohort, none of the discrepant results involved HSIL, CIN1, CIN2/3 or cancer samples. The adenocarcinoma sample that was negative for $14 \mathrm{hr}-\mathrm{HPVs}$ by both methods, harbored HPV44, 81, which are 1r-HPV types and not considered carcinogenic. Due to type-specific amplification, the NGS assay was able to detect a range of 2-12 HPV coinfections in 146/274 of samples, allowing unbiased analysis of existing HPV types in each sample.

Earlier studies have shown evidence of association between hr-HPVs and non-HPV STIs (including commensal STIs) with cervical carcinogenesis [17-26, 31]. In this study, 52.1\% samples in the 274-sample cohort harbored non-HPV STIs, of which $87.8 \%$ were commensal infections of three species: M. hominis, U. urealyticum and U. parvum. Moreover, almost half of the samples carried co-infections of HPV and non-HPV STIs. Thus, a comprehensive assay detecting multiple HPV and non-HPV STIs in a single test, is a useful tool to diagnose and study the correlation of hr-HPV and non-HPV co-infections. It is worth mentioning that the 14 non-HPV STIs in the NGS assay have different pathogenic characteristics and some of them are considered commensal species of the genital tract (such as M. hominis, U. urealyticum and U. paroum), existing in both healthy and symptomatic individuals [32]. 
HPV genotyping has been mostly focused on HPV16 and HPV18 due to their high prevalence in cervical cancer, although, other hr-types indicate high positive predictive value (PPV) $[8,10]$. Earlier studies suggested that extended HPV genotyping provides a better risk stratification and identification of women at increased risk of cervical cancer by simply providing individual risk assessment of HPV positive women [33-35]. The results of the two cohorts in this study show that co-infections of multiple HPVs and non-HPV STIs are widespread (Table 5). Due to limitation of current widely used assays, many of those STIs can go undetected or more than one assay might be needed.

It has been reported that non-transient hr-HPV infections and high viral loads is a risk marker for dysplasia and carcinoma in situ in normal cytology [11-16]. Such studies have been typically focused on few high-risk types. The NGS assay uses type-specific HPV primers for amplification and generates quantification information for each type in the panel. The wide spectrum of STIs and their quantification information could be useful for studying the association between STIs and diseases.

There are several NGS-based HPV assays [36-38] that commonly use more than one round of PCR. ChapterDx HPV-STI NGS assay uses one-step amplification workflow, which significantly reduces time/labor, the risk of cross contamination and allows easier automation.

\section{Conclusion}

ChapterDx HPV-STI NGS Assay is cost-effective as it detects a much broader spectrum of HPV/STI than other commercial kits, at a similar or lower cost per sample, with proven analytical performance; therefore, it is a valuable tool for large epidemiology and surveillance studies. Once evaluated with larger, well-characterized cohorts for clinical validation, this assay could also be applied to cervical cancer screening programs.

\section{Ethics approval and consent to participate}

This cohort is part of a screening study that is being carried out by the INEI-ANLIS Malbrán with the collaboration of the "Prof. A. Posadas" Hospital (Argentina); it was approved by the Research Ethics Board and the Ethics Committee of this hospital. Written informed consent was obtained before enrolling the participant women, and confidentiality and anonymity were guaranteed. 


\section{Competing interests}

Z.M., B.G., and C.W. are shareholders and employees of Chapter Diagnostics. A patent has been issued for the technology. The INEI-ANLIS Malbrán investigators declare no competing interests; they did not receive any financial contribution from Chapter Diagnostics.

\section{Authors' contributions}

Z.M., B.G., and C.W. are involved in the overall study of this assay and manuscript writing. X.C. and M. Li participated in the LoD assay and manuscript writing. The INEI-ANLIS Malbrán investigators are responsible for the selection of clinical samples previously characterized, participate in the analysis, interpretation of the data and revision of the manuscript.

\section{References}

1. (WHO) WHO: Global health sector strategy on sexually transmitted infections, 2016-2021. 2018.

2. Eisinger RW, Erbelding E, Fauci AS: Refocusing Research on Sexually Transmitted Infections. J Infect Dis 2020, 222(9):1432-1434.

3. Bernard HU, Burk RD, Chen Z, van Doorslaer K, zur Hausen H, de Villiers EM: Classification of papillomaviruses (PVs) based on $189 \mathrm{PV}$ types and proposal of taxonomic amendments. Virology 2010, 401(1):70-79.

4. Humans IWGotEoCRt: Biological agents. Volume $\mathbf{1 0 0}$ B. A review of human carcinogens. IARC Monogr Eval Carcinog Risks Hum 2012, 100(Pt B):1-441.

5. Munoz N, Bosch FX, de Sanjose S, Herrero R, Castellsague X, Shah KV, Snijders PJ, Meijer CJ, International Agency for Research on Cancer Multicenter Cervical Cancer Study G: Epidemiologic classification of human papillomavirus types associated with cervical cancer. $N$ Engl J Med 2003, 348(6):518-527.

6. Munoz N, Bosch FX, de Sanjose S, Tafur L, Izarzugaza I, Gili M, Viladiu P, Navarro $\mathrm{C}$, Martos $\mathrm{C}$, Ascunce $\mathrm{N}$ et al: The causal link between human papillomavirus and invasive cervical cancer: a population-based case-control study in Colombia and Spain. Int J Cancer 1992, 52(5):743-749.

7. Walboomers JM, Jacobs MV, Manos MM, Bosch FX, Kummer JA, Shah KV, Snijders PJ, Peto J, Meijer CJ, Munoz N: Human papillomavirus is a necessary cause of invasive cervical cancer worldwide. J Pathol 1999, 189(1):12-19.

8. Cuzick J, Wheeler C: Need for expanded HPV genotyping for cervical screening. Papillomavirus Res 2016, 2:112-115. 
medRxiv preprint doi: https://doi.org/10.1101/2021.11.24.21266783; this version posted November 26, 2021. The copyright holder for this preprint (which was not certified by peer review) is the author/funder, who has granted medRxiv a license to display the preprint in perpetuity.

It is made available under a CC-BY-ND 4.0 International license .

9. Joura EA, Ault KA, Bosch FX, Brown D, Cuzick J, Ferris D, Garland SM, Giuliano AR, Hernandez-Avila M, Huh W et al: Attribution of 12 high-risk human papillomavirus genotypes to infection and cervical disease. Cancer Epidemiol Biomarkers Prev 2014, 23(10):1997-2008.

10. Wright TC, Jr., Parvu V, Stoler MH, Kodsi S, Eckert K, Yanson K, Cooper CK: HPV infections and cytologic abnormalities in vaccinated women 21-34years of age: Results from the baseline phase of the Onclarity trial. Gynecol Oncol 2019, 153(2):259-265.

11. Trevisan A, Schlecht NF, Ramanakumar AV, Villa LL, Franco EL, Ludwig-McGill Study G: Human papillomavirus type 16 viral load measurement as a predictor of infection clearance. J Gen Virol 2013, 94(Pt 8):1850-1857.

12. Moberg M, Gustavsson I, Gyllensten U: Type-specific associations of human papillomavirus load with risk of developing cervical carcinoma in situ. Int J Cancer 2004, 112(5):854-859.

13. Moberg M, Gustavsson I, Wilander E, Gyllensten U: High viral loads of human papillomavirus predict risk of invasive cervical carcinoma. Br J Cancer 2005, 92(5):891-894.

14. Swan DC, Tucker RA, Tortolero-Luna G, Mitchell MF, Wideroff L, Unger ER, Nisenbaum RA, Reeves WC, Icenogle JP: Human papillomavirus (HPV) DNA copy number is dependent on grade of cervical disease and HPV type. J Clin Microbiol 1999, 37(4):1030-1034.

15. Xi LF, Hughes JP, Edelstein ZR, Kiviat NB, Koutsky LA, Mao C, Ho J, Schiffman M: Human Papillomavirus (HPV) type 16 and type 18 DNA Loads at Baseline and Persistence of Type-Specific Infection during a 2-year follow-up. J Infect Dis 2009, 200(11):1789-1797.

16. Xi LF, Hughes JP, Castle PE, Edelstein ZR, Wang C, Galloway DA, Koutsky LA, Kiviat NB, Schiffman M: Viral load in the natural history of human papillomavirus type 16 infection: a nested case-control study. J Infect Dis 2011, 203(10):1425-1433.

17. de Abreu AL, Malaguti N, Souza RP, Uchimura NS, Ferreira EC, Pereira MW, Carvalho MD, Pelloso SM, Bonini MG, Gimenes F et al: Association of human papillomavirus, Neisseria gonorrhoeae and Chlamydia trachomatis coinfections on the risk of high-grade squamous intraepithelial cervical lesion. Am J Cancer Res 2016, 6(6):1371-1383.

18. Verteramo R, Pierangeli A, Mancini E, Calzolari E, Bucci M, Osborn J, Nicosia R, Chiarini F, Antonelli G, Degener AM: Human Papillomaviruses and genital coinfections in gynaecological outpatients. BMC Infect Dis 2009, 9:16.

19. Biernat-Sudolska M, Szostek S, Rojek-Zakrzewska D, Klimek M, Kosz-Vnenchak $\mathrm{M}$ : Concomitant infections with human papillomavirus and various 
mycoplasma and ureaplasma species in women with abnormal cervical cytology. Adv Med Sci 2011, 56(2):299-303.

20. Discacciati MG, Gimenes F, Pennacchi PC, Faiao-Flores F, Zeferino LC, Derchain SM, Teixeira JC, Costa MC, Zonta M, Termini L et al: MMP-9/RECK Imbalance: A Mechanism Associated with High-Grade Cervical Lesions and Genital Infection by Human Papillomavirus and Chlamydia trachomatis. Cancer Epidemiol Biomarkers Prev 2015, 24(10):1539-1547.

21. Finan RR, Musharrafieh U, Almawi WY: Detection of Chlamydia trachomatis and herpes simplex virus type 1 or 2 in cervical samples in human papilloma virus (HPV)-positive and HPV-negative women. Clin Microbiol Infect 2006, 12(9):927-930.

22. Paba P, Bonifacio D, Di Bonito L, Ombres D, Favalli C, Syrjanen K, Ciotti M: Coexpression of HSV2 and Chlamydia trachomatis in HPV-positive cervical cancer and cervical intraepithelial neoplasia lesions is associated with aberrations in key intracellular pathways. Intervirology 2008, 51(4):230-234.

23. Samoff E, Koumans EH, Markowitz LE, Sternberg M, Sawyer MK, Swan D, Papp JR, Black CM, Unger ER: Association of Chlamydia trachomatis with persistence of high-risk types of human papillomavirus in a cohort of female adolescents. Am J Epidemiol 2005, 162(7):668-675.

24. Smith JS, Bosetti C, Munoz N, Herrero R, Bosch FX, Eluf-Neto J, Meijer CJ, Van Den Brule AJ, Franceschi S, Peeling RW et al: Chlamydia trachomatis and invasive cervical cancer: a pooled analysis of the IARC multicentric case-control study. Int J Cancer 2004, 111(3):431-439.

25. Smith JS, Herrero R, Bosetti C, Munoz N, Bosch FX, Eluf-Neto J, Castellsague X, Meijer CJ, Van den Brule AJ, Franceschi $S$ et al: Herpes simplex virus-2 as a human papillomavirus cofactor in the etiology of invasive cervical cancer. J Natl Cancer Inst 2002, 94(21):1604-1613.

26. Zhang ZF, Graham S, Yu SZ, Marshall J, Zielezny M, Chen YX, Sun M, Tang SL, Liao CS, Xu JL et al: Trichomonas vaginalis and cervical cancer. A prospective study in China. Ann Epidemiol 1995, 5(4):325-332.

27. FDA: Policy for Coronavirus Disease-2019 Tests During the Public Health Emergency (Revised), U.S. Department of Health and Human Services. Food and Drug Administration (FDA). https://wwwfdagov/media/135659/download 2020.

28. Eklund C, Forslund O, Wallin KL, Dillner J: Global improvement in genotyping of human papillomavirus DNA: the 2011 HPV LabNet International Proficiency Study. J Clin Microbiol 2014, 52(2):449-459.

29. van den Brule AJ, Pol R, Fransen-Daalmeijer N, Schouls LM, Meijer CJ, Snijders PJ: GP5+/6+ PCR followed by reverse line blot analysis enables rapid and highthroughput identification of human papillomavirus genotypes. J Clin Microbiol 2002, 40(3):779-787. 
30. Meijer CJ, Berkhof J, Castle PE, Hesselink AT, Franco EL, Ronco G, Arbyn M, Bosch FX, Cuzick J, Dillner J et al: Guidelines for human papillomavirus DNA test requirements for primary cervical cancer screening in women 30 years and older. Int J Cancer 2009, 124(3):516-520.

31. Wallin KL, Wiklund F, Luostarinen T, Angstrom T, Anttila T, Bergman F, Hallmans G, Ikaheimo I, Koskela P, Lehtinen M et al: A population-based prospective study of Chlamydia trachomatis infection and cervical carcinoma. Int J Cancer 2002, 101(4):371-374.

32. Horner P, Donders G, Cusini M, Gomberg M, Jensen JS, Unemo M: Should we be testing for urogenital Mycoplasma hominis, Ureaplasma parvum and Ureaplasma urealyticum in men and women? - a position statement from the European STI Guidelines Editorial Board. J Eur Acad Dermatol Venereol 2018, 32(11):1845-1851.

33. Bottari F, Iacobone AD, Passerini R, Preti EP, Sandri MT, Cocuzza CE, Gary DS, Andrews JC: Human Papillomavirus Genotyping Compared With a Qualitative High-Risk Human Papillomavirus Test After Treatment of High-Grade Cervical Intraepithelial Neoplasia: A Systematic Review. Obstet Gynecol 2019, 134(3):452462.

34. Demarco M, Hyun N, Carter-Pokras O, Raine-Bennett TR, Cheung L, Chen X, Hammer A, Campos N, Kinney W, Gage JC et al: A study of type-specific HPV natural history and implications for contemporary cervical cancer screening programs. EClinicalMedicine 2020, 22:100293.

35. Jentschke M, Soergel P, Hillemanns P: Importance of HPV Genotyping for the Screening, Therapy and Management of Cervical Neoplasias. Geburtshilfe Frauenheilkd 2012, 72(6):507-512.

36. Ardhaoui M, Ennaifer E, De Matos Salim AC, Gomez FM, Laasili T, Boubaker MS, Guizani I: Nested PCR followed by NGS: Validation and application for HPV genotyping of Tunisian cervical samples. PLoS One 2021, 16(8):e0255914.

37. Arroyo Muhr LS, Lagheden C, Lei J, Eklund C, Nordqvist Kleppe S, Sparen P, Sundstrom K, Dillner J: Deep sequencing detects human papillomavirus (HPV) in cervical cancers negative for HPV by PCR. Br J Cancer 2020, 123(12):1790-1795.

38. Wagner S, Roberson D, Boland J, Yeager M, Cullen M, Mirabello L, Dunn ST, Walker J, Zuna R, Schiffman $M$ et al: Development of the TypeSeq Assay for Detection of 51 Human Papillomavirus Genotypes by Next-Generation Sequencing. J Clin Microbiol 2019, 57(5). 
medRxiv preprint doi: https://doi.org/10.1101/2021.11.24.21266783; this version posted November 26, 2021. The copyright holder for this preprint (which was not certified by peer review) is the author/funder, who has granted medRxiv a license to display the preprint in perpetuity.

It is made available under a CC-BY-ND 4.0 International license .

Fig. 1. A schematic illustration of ChapterDx HPV-STI NGS workflow

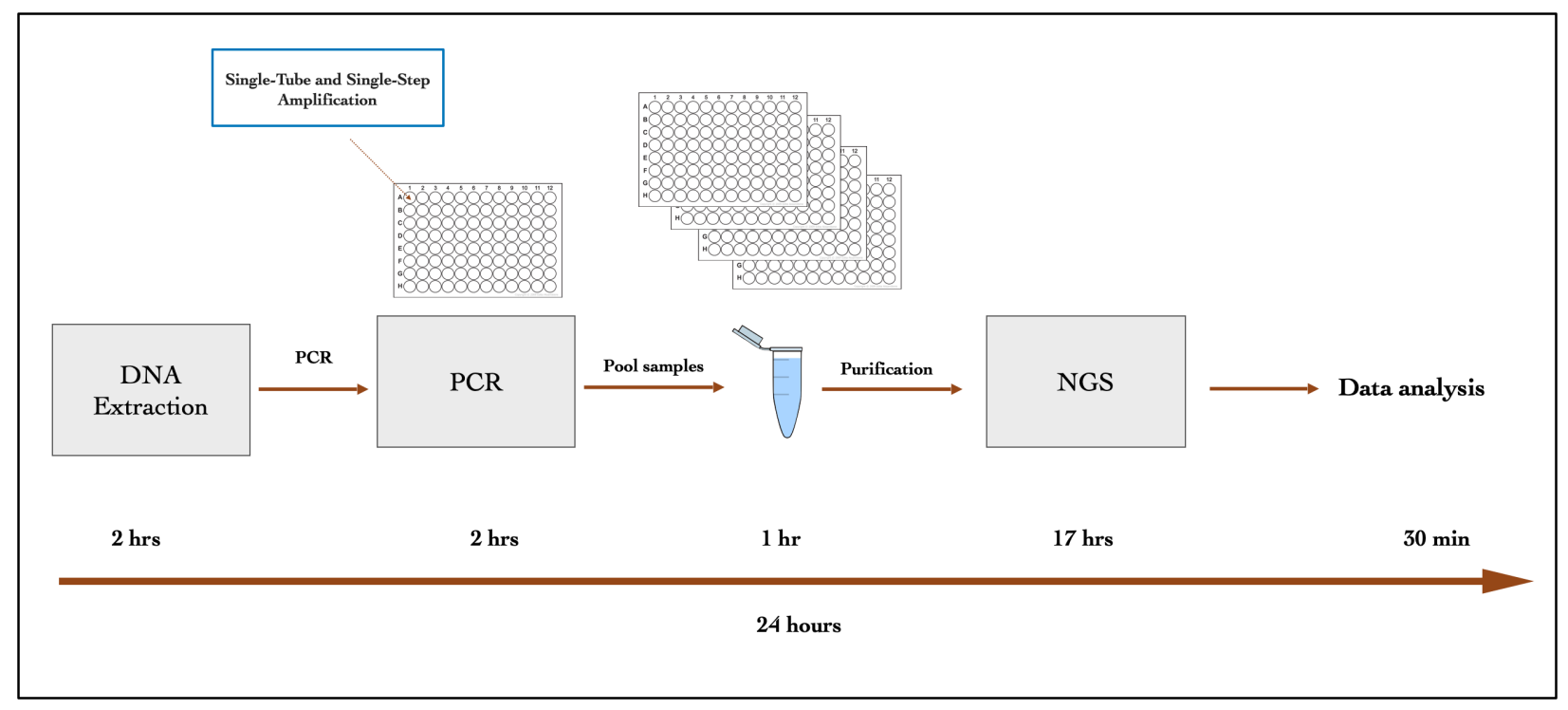


Table 1. The list of $43^{*}$ HPV/STI in the NGS panel

\begin{tabular}{|l|l|l|}
\hline \multirow{3}{*}{19 HR-HPV Types } & \multicolumn{2}{|l|}{ HPV-16, 18, 26, 31, 33, 35, 39, 45, 51, 52, 53, 56, 58, 59, 66, 68a, 68b, 73, 82 } \\
\hline \multirow{5}{*}{10 LR-HPV Types } & HPV-6, 11, 40, 42, 43, 44, 55, 61, 81, 83 \\
\hline \multirow{5}{*}{ 14 STI Species/Types } & Chlamydia trachomatis (serovars A to K) & Ureaplasma urealyticum \\
\cline { 2 - 3 } & Neisseria gonorrhoeae & Ureaplasma parvum \\
\cline { 2 - 3 } & Mycoplasma genitalium & Herpes simplex virus type 1 \\
\cline { 2 - 3 } & Trichomonas vaginalis & Herpes simplex virus type 2 \\
\cline { 2 - 3 } & Treponema pallidum & Varicella zoster virus \\
\cline { 2 - 3 } & Haemophilus ducreyi & Chlamydia trachomatis serovar L1 \\
\cline { 2 - 3 } & Mycoplasma hominis & Chlamdia trachomatis serovar L2 \\
\hline
\end{tabular}

${ }^{*}$ For simplicity, HPV68a and HPV68b are listed as separate HPV types; Chlamydia trachomatis serovar L1 and L2 are listed as separate STIs. 
Table 2a. LoD results of 43 control fragment pools for 23 replicates of 100 copies and 24 replicates of 50 and 20 copies

\begin{tabular}{|c|c|c|c|}
\hline Copy Number (43 control Pool) & Type/Species & Replicate Detection Rate & \% LoD Detection \\
\hline 100 Copies & $43 / 43$ & $24 / 24$ & $100 \%$ \\
\hline 50 Copies & $43 / 43$ & $24 / 24$ & $100 \%$ \\
\hline \multirow{3}{*}{20 Copies } & $34 / 43$ & $24 / 24$ & $100 \%$ \\
\cline { 2 - 4 } & $8 / 43$ & $23 / 24$ & $95.8 \%$ \\
\cline { 2 - 4 } & $1 / 43$ & $21 / 24$ & $87.5 \%$ \\
\hline
\end{tabular}

Table 2b. 20 copies LoD for 9 control fragments below $100 \%$ agreement

\begin{tabular}{|l|c|c|}
\hline \multicolumn{1}{|c|}{ Species/Types } & Replicate agreement & LoD agreement \\
\hline HPV6 & $23 / 24$ & $96 \%$ \\
\hline HPV11 & $23 / 24$ & $96 \%$ \\
\hline HPV39 & $23 / 24$ & $96 \%$ \\
\hline HPV42 & $23 / 24$ & $96 \%$ \\
\hline HPV43 & $23 / 24$ & $96 \%$ \\
\hline HPV45 & $23 / 24$ & $96 \%$ \\
\hline HPV56 & $23 / 24$ & $96 \%$ \\
\hline HPV83 & $23 / 24$ & $96 \%$ \\
\hline M. Genitalium & $21 / 24$ & $88 \%$ \\
\hline
\end{tabular}


Table 3. Overall concordance of 274 clinical samples between ChapterDx HPV-STI NGS assay and Roche cobas for 14 high-risk HPVs, stratified by histology and cytology

\begin{tabular}{|c|c|c|c|c|c|c|}
\hline \multirow{12}{*}{$\begin{array}{c}\text { ChapterDx HPV-STI } \\
\text { NGS Assay }\end{array}$} & \multicolumn{6}{|c|}{ Roche cobas 4800} \\
\hline & & & Negative & Positive & Agreement & kappa ${ }^{a}$ \\
\hline & \multirow{2}{*}{ Overall Agreement } & Negative & 26 & 0 & \multirow{2}{*}{$97.5 \%$} & \multirow{2}{*}{0.867 (95\% CI 0.771-0.963) } \\
\hline & & Positive & 7 & 241 & & \\
\hline & \multirow{2}{*}{ CIN2/3 (59) } & Negative & 0 & 0 & \multirow{2}{*}{$100 \%$} & \multirow{2}{*}{1} \\
\hline & & Positive & 0 & 59 & & \\
\hline & \multirow{2}{*}{ HSIL (57) } & Negative & 0 & 0 & \multirow{2}{*}{$100 \%$} & \multirow{2}{*}{1} \\
\hline & & Positive & 0 & 57 & & \\
\hline & \multirow{2}{*}{ Squamous carcinoma $^{\mathrm{b}}(3)$} & Negative & 1 & 0 & \multirow{2}{*}{$100 \%$} & \multirow{2}{*}{1} \\
\hline & & Positive & 0 & 2 & & \\
\hline & \multirow{2}{*}{ Adenocarcinoma $^{c}(2)$} & Negative & 1 & 0 & \multirow{2}{*}{$100 \%$} & \multirow{2}{*}{1} \\
\hline & & Positive & 0 & 1 & & \\
\hline
\end{tabular}

aInterpretation values: poor $(<0.20)$, fair $(0.21-0.40)$, moderate $(0.41-0.60)$, good $(0.61-0.80)$, very good $(0.81-1.00)$

${ }^{\mathrm{b}} \mathrm{The}$ HPV negative squamous carcinoma was negative with three methods.

${ }^{\mathrm{C}}$ The negative adenocarcinoma was negative by NGS and Roche assays for $14 \mathrm{HPV}$ types but was positive for HPV44 and 81 by ChapterDx HPV-STI NGS assay. 
Table 4a. Concordance between ChapterDx HPV-STI NGS assay and Roche cobas assay for 14 high-risk HPVs

\begin{tabular}{|c|c|c|c|c|c|c|c|}
\hline \multirow{11}{*}{$\begin{array}{c}\text { ChapterDx HPV-STI } \\
\text { NGS Assay }\end{array}$} & \multicolumn{7}{|c|}{ Roche cobas 4800} \\
\hline & & & Negative & Positive & Total & Agreement & kappa \\
\hline & \multirow{3}{*}{ HPV16 } & Negative & 183 & 1 & 182 & \multirow{3}{*}{$98.5 \%$} & \multirow{3}{*}{0.967 (95\% CI $0.934-0.999)$} \\
\hline & & Positive & 3 & 87 & 92 & & \\
\hline & & Total & 184 & 90 & 274 & & \\
\hline & \multirow{3}{*}{ HPV18 } & Negative & 250 & 3 & 253 & \multirow{3}{*}{$98.5 \%$} & \multirow{3}{*}{0.901 (95\% CI $0.805-0.997)$} \\
\hline & & Positive & 1 & 20 & 21 & & \\
\hline & & Total & 251 & 23 & 274 & & \\
\hline & \multirow{3}{*}{$\begin{array}{c}\text { Others }^{\mathrm{b}} \\
\text { (12 HR Types) }\end{array}$} & Negative & 79 & 7 & 86 & \multirow{3}{*}{$96.0 \%$} & \multirow{3}{*}{0.906 (95\% CI 0.851-0.960) } \\
\hline & & Positive & 4 & 184 & 188 & & \\
\hline & & Total & 83 & 191 & 274 & & \\
\hline
\end{tabular}

anterpretation values: poor $(<0.20)$, fair $(0.21-0.40)$, moderate $(0.41-0.60)$, good $(0.61-0.80)$, very good $(0.81-1.00)$; ${ }^{6} \mathrm{HPV} 31,33,35,39,45,51,52,56,58,59,66$, and 68

Table $4 b$. Nr of 2 to 12 HPV co-infections in 274 samples

\begin{tabular}{|l|c|c|c|c|c|c|c|c|c|c|c|}
\hline Nr of HPV co-infections & 2 & 3 & 4 & 5 & 6 & 7 & 8 & 9 & 10 & 12 & total \\
\hline Nr of specimens & 67 & 29 & 20 & 19 & 5 & 1 & 1 & 2 & 1 & 1 & 146 \\
\hline
\end{tabular}


Table 5a. Other STIs detected in the 274 samples by ChapterDx HPV-STI NGS assay

\begin{tabular}{|l|c|}
\hline \multicolumn{1}{|c|}{ STIs detected } & $\mathrm{Nr} / \%$ \\
\hline Chlamydia trachomatis & $4(1.5 \%)$ \\
\hline Trichomonas vaginalis & $16(5.8 \%)$ \\
\hline Mycoplasma genitalium & $1(0.36 \%)$ \\
\hline Mycoplasma hominis & $69(25.2 \%)$ \\
\hline Ureaplasma parvum & $88(32.1 \%)$ \\
\hline Ureaplasma urealyticum & $23(8.4 \%)$ \\
\hline Herpes simplex virus type 2 & $4(1.5 \%)$ \\
\hline
\end{tabular}

Table 5b. Nr of 2 to 4 non-HPV STI co-infections in 274 samples

\begin{tabular}{|l|c|c|c|c|}
\hline Nr of STI co-infections & 2 & 3 & 4 & Total \\
\hline Nr of specimens & 45 & 6 & 1 & 52 \\
\hline
\end{tabular}


Table 6. Comparison of ChapterDX HPV-STI NGS and Roche cobas for samples with combined HPV and STI co-infection

\begin{tabular}{|c|l|l|}
\hline Sample & Roche Cobas & Chapter Diagnostics HPV-STI NGS Panel \\
\hline 1 & HPV16 and others & HPV16, 35, Trichomonas vaginalis, Mycoplasma hominis \\
\hline 2 & Others & HPV33, 56, 43, Trichomonas vaginalis, Mycoplasma hominis \\
\hline 3 & HPV16, 18, others & HPV16, 18, 31, 33, 45, 52, 56, 68a, 42, 61 Trichomonas vaginalis, Mycoplasma hominis \\
\hline 4 & Others & HPV58 and Chlamydia Trachomatis \\
\hline 5 & Others & HPV16, 39, and Herpes Simplex II (HSV2) \\
\hline 6 & Others & HPV66, 68a and Chlamydia Trachomatis \\
\hline 7 & Others & HPV31, 51, Mycoplasma genitalium, Trichomonas vaginalis, Mycoplasma hominis, Ureaplasma Parvum \\
\hline 8 & HPV16 and others & HPV16, 45, 11, 81, 83, Herpes Simplex II (HSV2), Mycoplasma hominis, Trichomonas vaginalis \\
\hline 9 & Others & HPV58, 42, Chlamydia trachomatis \\
\hline
\end{tabular}


Supplementary Table S1. Comparison of Illumina sequencing platforms and their respective sequencing kits for HPV-STI analysis

\begin{tabular}{|l|l|c|c|c|c|}
\hline Illumina Platforms & Illumina Seq. Kit & Read Length & Output & Reads/Run & Samples \\
\hline iSeq 100 & iSeq 2 150 & $2 \times 150$ & $1.2 \mathrm{~Gb}$ & $4 \mathrm{M}$ & 192 \\
\hline MiniSeq & Mid-Output Kit & $2 \times 150$ & $2.4 \mathrm{~Gb}$ & $8 \mathrm{M}$ & 384 \\
\hline MiniSeq & High-Output Kit & $2 \times 150$ & $7.5 \mathrm{~Gb}$ & $25 \mathrm{M}$ & 1,200 \\
\hline MiSeq & MiSeq v2 Nano & $2 \times 150$ & $300 \mathrm{Mb}$ & $1 \mathrm{M}$ & 48 \\
\hline MiSeq & MiSeq v2 Micro & $2 \times 150$ & $1.2 \mathrm{~Gb}$ & $4 \mathrm{M}$ & 192 \\
\hline MiSeq & MiSeq v2 & $2 \times 150$ & $4.5-5.1 \mathrm{~Gb}$ & $12-15 \mathrm{M}$ & $576-720$ \\
\hline NextSeq & Mid-Output Kit & $2 \times 150$ & $32.5-39 \mathrm{~Gb}$ & $130 \mathrm{M}$ & 6,240 \\
\hline NextSeq & High-Output Kit & $2 \times 150$ & $100-120 \mathrm{~Gb}$ & $400 \mathrm{M}$ & $>10,000$ \\
\hline
\end{tabular}

